\title{
Propuesta de una metodología para el estudio de competencia tecnológica, en empresas fabricantes de medicamentos
}

Proposal of a Methodology for the Study of Technological Competence in Pharmaceutical Manufacturing

\section{Proposta de uma metodologia para o estudo de competência tecnológica, em empresas fabricantes de medicamentos}

\author{
Bibiana Margarita Vallejo* \\ John Jairo Gallo* \\ Clara Eugenia Plazas ${ }^{* * *}$
}

Fecha recibido: 14 de diciembre de 2015

Fecha aprobado: 12 de febrero de 2016

Doi: http://dx.doi.org/10.12804/rev.univ.empresa.31.2016.01

Para citar este artículo: Vallejo, B., Gallo, J., \& Plazas, C. (2016). Propuesta de una metodología para el estudio de competencia tecnológica, en empresas fabricantes de medicamentos. Universidad \& Empresa, 18(31), 11 27. Doi: http://dx.doi.org/10.12804/rev.univ.empresa.31.2016.01

\section{RESUMEN}

Se identificaron ocho variables asociadas al concepto de competencia tecnológica para estudiar una empresa productora de medicamentos: integración de la cadena de abastecimiento primaria, evaluación de escala de producción, transferencia tecnológica, tecnologías de la información y la comunicación, perfil exportador, innovación y desarrollo, recursos humanos y sistemas de garantía y aseguramiento de calidad. Se estudió el comportamiento de cada

\footnotetext{
Doctora en Ingeniería Química. Universidad Nacional de Colombia. Departamento de Farmacia. Bogotá, Colombia. Correo electrónico: bmvallejod@unal.edu.co

** Magister en Ciencias Farmacéuticas. Pharmaceutical Supply Chain S.A.S. Bogotá, Colombia. Correo electrónico: johngalloqf@gmail.com

*** Doctora en Ingeniería - Ciencia y Tecnología de Materiales. Universidad Nacional de Colombia. Departamento de Farmacia. Bogotá, Colombia. Correo electrónico: ceplazasb@unal.edu.co
} 
una de las variables y cómo su relación permitiría establecer criterios de comparación entre empresas del mismo sector. Se aplicó una encuesta en 22 laboratorios de producción de medicamentos y los datos obtenidos fueron analizados a través de la estrategia metodológica propuesta.

Palabras clave: competencia tecnológica, desarrollo tecnológico, modelo descriptivo, producción de medicamentos.

\section{ABSTRACT}

As a starting point 8 variables that are associated with the concept of technological competence in a pharmaceutical production company were selected: supply chain integration, evaluation of scale production, technology transfer, information and communications technology, export profile, innovation and development, human resources and assurance systems and quality assurance. It was proposed to study the behavior of each of the variables and how their relationship would develop criteria for comparing companies in the same sector. Twenty two national pharmaceutical companies were selected, and a survey was applied and the data obtained were analyzed through a methodology proposed in this research.

Keywords: Technological competency, technological development, descriptive modeling, drug production.

\section{RESUMO}

Identificaram-se 8 variáveis associadas ao conceito de competência tecnológica para estudar uma empresa produtora de medicamentos: integração da cadeia de abastecimento primária, avaliação de escala de produção, transferência tecnológica, tecnologias da informação e a comunicação, perfil exportador, inovação e desenvolvimento de cada uma das variáveis e como a sua relação permitiria estabelecer critérios de comparação entre empresas do mesmo setor. Aplicou-se um inquérito em 22 laboratórios de produção de medicamentos e os dados obtidos foram analisados através da estratégia metodológica proposta.

Palavras-chave: Competência tecnológica, desenvolvimento tecnológico, modelo descritivo, produção de medicamentos. 
INTRODUCCIÓN

El estudio de la competencia tecnológica en el sector farmacéutico es una actividad compleja, debido a que no existen indicadores formalmente establecidos para su definición; en el caso de los procesos de producción de una empresa farmacéutica, son varios los componentes asociados al tipo de producto, denominado forma farmacéutica, su condición de esterilidad y las características farmacológicas de los ingredientes activos; todos ellos definen desde el proceso de producción las características asociadas a un determinado nivel de desarrollo tecnológico propio de cada organización productora.

Cuando se pretende establecer esta capacidad diferenciadora entre laboratorios fabricantes y productores de medicamentos, es posible plantearlo en términos de variables relacionadas con los procesos de manufactura como: integración de la cadena de abastecimiento primaria, evaluación de escala de producción, transferencia tecnológica, tecnologías de la información y la comunicación, perfil exportador, innovación y desarrollo, recursos humanos y sistemas de garantía y aseguramiento de calidad.

Estudiar el comportamiento de las organizaciones frente a estas variables, permite definir posiciones y perspectivas, observar comportamientos característicos que son reconocidos como de buen desempeño o altamente competitivos. En esta investigación se propone una metodología para evaluar las competencias tecnológicas asociadas al proceso de transformación de materiales, de laboratorios que fabrican medicamentos, analizando las principales variables que afectan su desempeño. El resultado de esta metodología es analizado en 22 laboratorios de producción de medicamentos en la ciudad de Bogotá.

\section{REVISIÓN DE LA LITERATURA}

El potencial para la manufactura de un bien tangible en una organización está relacionado con la disponibilidad de materias primas, personal y tecnología, que interactúan de forma definida cuidadosamente (Rodríguez, 2006); todo producto o bien tangible requiere: materias primas que adquieren valor al ser transformadas, equipos que realizan operaciones específicas, personal con unos conocimientos básicos y destrezas mínimas para ejecutar su labor y un lugar o espacio en el cual se desarrolla la actividad productiva; además de todos los recursos adicionales fundamentales para cualquier operación de manufactura (electricidad, agua, alcantarillado,comunicaciones, vías de transporte) (Attridge \& Preker, 
2005). A este potencial para transformar un conjunto de materiales en un producto se le denomina "Capacidad de Manufactura".

En un proceso de transformación de materiales para la fabricación de medicamentos se lleva a cabo una secuencia lógica de operaciones acompañadas de un flujo permanente de información, proceso durante el cual los insumos adquieren un valor agregado frente a su condición inicial, ver figura 1 .

Figura 1. El proceso de transformación de materiales en el Sector Farmacéutico Industrial Productor (SFIP)

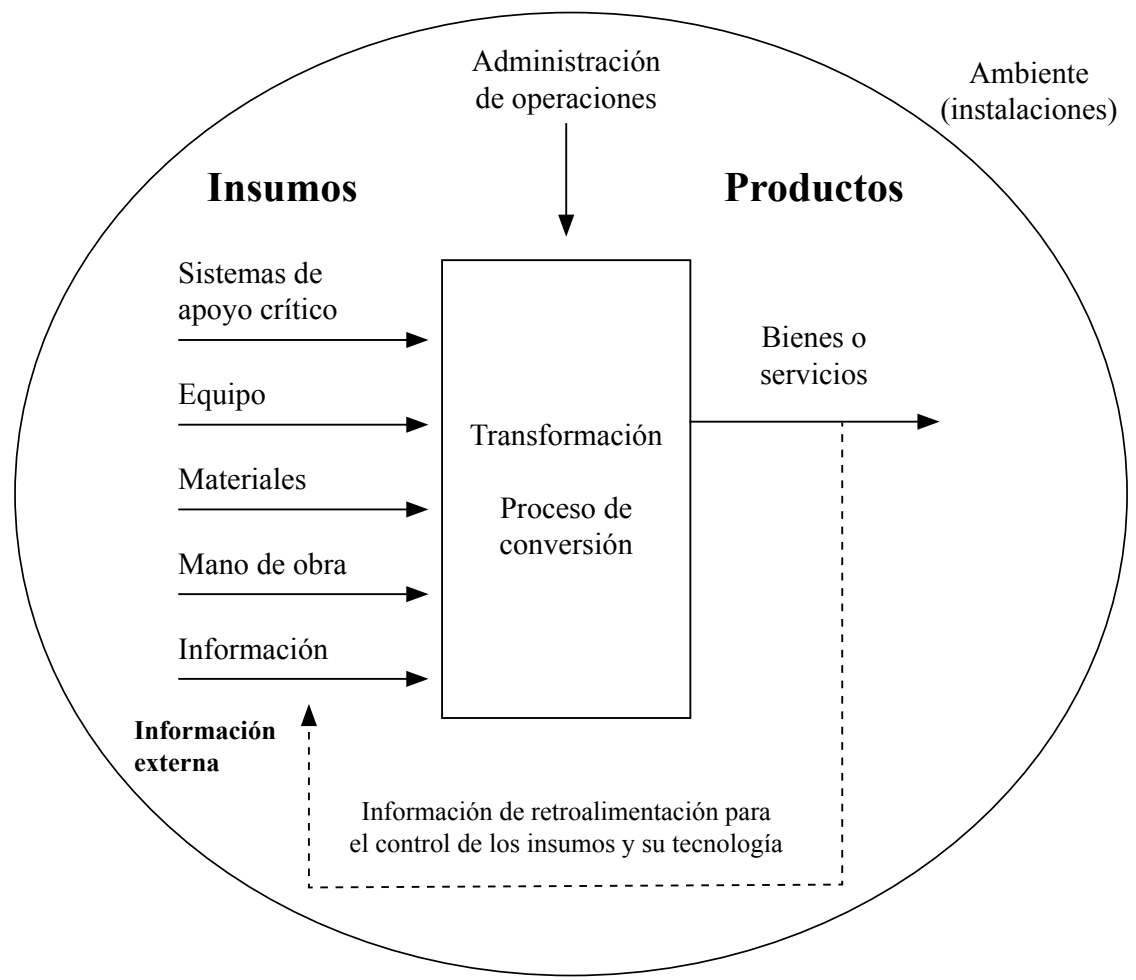

Fuente: Vallejo y Torres, 2007.

Los medicamentos corresponden a involucran sus procesos de producun grupo de bienes definidos como ción y las consecuencias fatales que de primera necesidad para la po- ocasionarían los errores durante su blación y debido a los riesgos que manufactura, requieren el cumpli- 
miento de normas estrictas de fabricación que se reúnen en el compendio denominado Buenas Prácticas de Manufactura (OMS, 1999).

Un medicamento presenta características asociadas a su proceso de elaboración, que diferencian la tecnología que puede ser empleada, entre estas se destacan el o los principios activos incorporados, la forma farmacéutica, la vía de administración y la concentración en la cual se encuentra el principio activo en la forma farmacéutica (Ministerio de la Protección Social, 2008).

De la misma forma en que cada medicamento tiene un conjunto de características que lo identifican de manera inequívoca, también se asocian particularidades específicas del proceso productivo, que en este estudio se identifica como "capacidad de manufactura" y que es evaluada en las organizaciones del SFIP. Se busca así reconocer denominadores comunes en los requisitos tecnológicos que demanda cada tipo de medicamento para ser manufacturado, respecto a materiales, instalaciones, métodos de trabajo y calidad y cantidad de personal (Gallo, Vallejo \& Plazas, 2010).

Con el interés por establecer una metodología que permita evaluar la competencia tecnológica de una empresa productora de medicamentos; se entiende que este concepto corresponde con la habilidad de una organización, para administrar sus procesos tecnológicos de manera eficiente, de forma que pueda desarrollar sus procesos productivos competitivamente (Gallo, 2010).

Se ha identificado que algunas variables pueden ser útiles para evaluar las competencias tecnológicas de un laboratorio productor de medicamentos así como su efecto sobre los procesos productivos de la empresa. En la tabla 1 se consolida el listado de las variables seleccionadas, las cuales en diferentes investigaciones han mostrado ser relevantes en la identificación de competencias tecnológicas.

Tabla 1. Variables para el estudio de competencias tecnológicas

\begin{tabular}{|l|l|}
\hline \multicolumn{1}{|c|}{$\begin{array}{c}\text { Variable a } \\
\text { evaluar }\end{array}$} & \multicolumn{1}{|c|}{ Importancia en el estudio } \\
\hline $\begin{array}{l}\text { Integración de } \\
\text { la cadena de } \\
\text { abastecimiento } \\
\text { primaria (ICAP) }\end{array}$ & $\begin{array}{l}\text { Define el grado de integración vertical de la organización y la eficiencia para } \\
\text { administrar la selección de proveedores y la gestión de compras (González, } \\
\text { 2004; Kaplan \& Laing, 2005; Meijboom \& Obel, 2007; WHO, 2007; Ala, } \\
\text { 2013). }\end{array}$ \\
\hline
\end{tabular}

Continúa 


\begin{tabular}{|c|c|}
\hline $\begin{array}{l}\text { Variable a } \\
\text { evaluar }\end{array}$ & Importancia en el estudio \\
\hline $\begin{array}{l}\text { Escala de } \\
\text { Producción (EP) }\end{array}$ & $\begin{array}{l}\text { Evalúa la eficiencia del proceso productivo relacionando el volumen de pro- } \\
\text { ducción que se puede manufacturar por lote. Se valora la relación entre la } \\
\text { infraestructura instalada y la infraestructura utilizada. Se puede identificar el } \\
\text { grado de diversificación de la organización y confrontarlo con su perfil pro- } \\
\text { ductor (Meijboom y Obel, 2007; Ryan y Shanebrook, 2004; Bessen, 2009). }\end{array}$ \\
\hline $\begin{array}{l}\text { Transferencia } \\
\text { Tecnológica } \\
\text { (TT) }\end{array}$ & $\begin{array}{l}\text { Estudia el perfil de adquisición y asimilación tecnológica en los últimos cinco } \\
\text { años. El comportamiento respecto a esta variable puede indicar parámetros de } \\
\text { evolución, crecimiento, retracción, concentración y/o especialización. Se eva- } \\
\text { lúa para las operaciones de producción y las operaciones de control de calidad } \\
\text { de manera independiente (Keld, 1996; Simango, 2000; Mora, 2003; Zucker } \\
\text { y Darby, 1997; Achilladelis y Antonakis, 2001; Departamento Nacional de } \\
\text { Planeación, 2008; Gnyawali y Park, 2009). }\end{array}$ \\
\hline $\begin{array}{l}\text { Tecnologías de } \\
\text { la información y } \\
\text { la comunicación } \\
\text { (TICS) }\end{array}$ & $\begin{array}{l}\text { Identifica los mecanismos diseñados para administrar la información al interior } \\
\text { de la organización. Establecimiento del grado de apropiación de las nuevas } \\
\text { herramientas informáticas diseñadas para realizar de manera eficiente la admi- } \\
\text { nistración de la información generada durante el proceso productivo, desde la } \\
\text { identificación de la necesidad del producto hasta su distribución (Albis, 2006; } \\
\text { Cohan, 1999). }\end{array}$ \\
\hline $\begin{array}{l}\text { Perfil exportador } \\
(\mathrm{PE})\end{array}$ & $\begin{array}{l}\text { Evalúa la exposición de los productos de la organización a mercados interna- } \\
\text { cionales, permite reconocer la competitividad de los productos de una organi- } \\
\text { zación frente a compañías presentes en mercados externos; se evidencia el tipo } \\
\text { de productos para los cuales existe presencia en mercados internacionales } \\
\text { y en qué aspectos se puede establecer un alto nivel de competitividad (Ryan y } \\
\text { Shanebrook, 2004; Cohan, 1999). }\end{array}$ \\
\hline $\begin{array}{l}\text { Innov } \\
\text { Desar }\end{array}$ & $\begin{array}{l}\text { Evalúa la directriz de la compañía en el desarrollo y lanzamiento de produc- } \\
\text { tos al mercado en los últimos cinco años. Los resultados permiten identificar } \\
\text { una tendencia de la producción farmacéutica en relación con la importación } \\
\text { de productos terminados, a granel o semielaborados. Adicionalmente, se es- } \\
\text { tablece el perfil innovador o generador de nuevos productos que maneja la } \\
\text { organización (WHO, 2007; Ryan y Shanebrook, 2004; Keld, 1996; Simango, } \\
\text { 2000; Cohan, 1999). }\end{array}$ \\
\hline $\begin{array}{l}\text { Recursos } \\
\text { Humanos (RH) }\end{array}$ & $\begin{array}{l}\text { Identifica los procesos de selección de personal calificado, aspecto que afecta } \\
\text { drásticamente la competitividad de las organizaciones. Reconocer el perfil de } \\
\text { formación del personal que labora en las organizaciones productoras de medi- } \\
\text { camentos puede indicar una fortaleza o una debilidad (Simango, 2000; Kaplan } \\
\text { y Laing, 2005; Albis, 2006; De Carolis, 2003). }\end{array}$ \\
\hline $\begin{array}{l}\text { Sistemas de } \\
\text { Garantía y } \\
\text { Aseguramiento } \\
\text { de la Calidad } \\
\text { (SGAC) }\end{array}$ & $\begin{array}{l}\text { Identifica la capacidad para adoptar reglamentación de estándares internacio- } \\
\text { nales y proyectar la organización para competir en mercados internacionales. } \\
\text { Se puede evidenciar cuáles sistemas de garantía de calidad ha adoptado cada } \\
\text { organización y cuáles están en proyecto de implementación. Cabe destacar que } \\
\text { las organizaciones farmacéuticas deben cumplir estrictos sistemas de control } \\
\text { (WHO, 2007; Ryan y Shanebrook, 2004). }\end{array}$ \\
\hline
\end{tabular}

Fuente: elaboración propia. 


\section{METODOLOGÍA}

Con el fin de llevar a cabo la evaluación de las competencias tecnológicas en empresas fabricantes de medicamentos, se utilizaron cada una de las variables descritas previamente definiendo para cada una de ellas los criterios asociados a un comportamiento de alta competencia, ver tabla 2.

Tabla 2. Comportamientos de mayor competitividad por cada variable de estudio para empresas productoras de medicamentos

\begin{tabular}{|c|c|}
\hline Variable & Comportamiento de alta competencia \\
\hline ICAP & $\begin{array}{l}\text { La organización ha fortalecido su relación con los proveedores por medio de } \\
\text { fenómenos de fusión, adquisición o elaboración de contratos que aseguran el } \\
\text { abastecimiento de los insumos. }\end{array}$ \\
\hline EP & $\begin{array}{l}\text { La organización ha desarrollado escalas de producción para responder a grandes } \\
\text { mercados disminuyendo los costos fijos de producción. }\end{array}$ \\
\hline TT & $\begin{array}{l}\text { La organización ha realizado procesos de transferencia, adecuación o desarrollo } \\
\text { de tecnología en sus procesos productivos en los últimos cinco años }\end{array}$ \\
\hline TICS & $\begin{array}{l}\text { La organización ha optimizado el manejo de la información en cada una de las } \\
\text { operaciones que desarrolla y ha mejorado el flujo a lo largo del proceso productivo. }\end{array}$ \\
\hline $\mathrm{PE}$ & La organización ha mejorado su perfil exportador accediendo a nuevos mercados. \\
\hline ID & $\begin{array}{l}\text { La organización ha fortalecido sus procesos de diseño y desarrollo a través } \\
\text { del lanzamiento de nuevos productos al mercado. }\end{array}$ \\
\hline $\mathrm{RH}$ & $\begin{array}{l}\text { La organización cuenta con personal suficiente y calificado, con bajos niveles } \\
\text { de rotación y procesos eficientes de selección. }\end{array}$ \\
\hline SGAC & $\begin{array}{l}\text { La organización tiene la capacidad para cumplir con estándares de calidad } \\
\text { relacionados con producto, proceso y personal. }\end{array}$ \\
\hline
\end{tabular}

Fuente: elaboración propia.

A continuación se diseñó un instrumento de captura de información, compuesto por preguntas con respuesta cerradas, que no dieran lugar a interpretaciones subjetivas por el encuestado y se utilizó una escala definida para la cuantificación. El instrumento de captura de información (ICI) fue diseñado para ser empleado en una encuesta realizada por un profesional Químico Farmacéutico en una entrevista personalizada con un director técnico.

Para la valoración numérica de las posibles respuestas, se construyó una matriz de puntaje que permitió la conversión de datos categóricos cualitativos, en datos categóricos cuantitativos. 
Para establecer el grado de competencia tecnológica de una organización, se propuso que los resultados obtenidos para cada variable y su operación matemática, estas pueden ser comparados con un supuesto teórico definido durante la investigación como el mejor comportamiento. A partir de los puntajes más altos que se alcancen con la aplicación del ICI, es posible establecer la situación de una organización en particular frente a un referente o también describir el comportamiento del sector en un instante determinado.

En el estudio sobre la pertinencia de la metodología propuesta para la evaluación de competencias tecnológicas, se elaboró una base de datos de los laboratorios con certificación en Buenas Prácticas de Manufactura en un período de dos años; la información fue suministrada por el Invima, identificando las autorizaciones de fabricación en función de la infraestructura, con la cual se estableció un "perfil productor" de cada organización.

A partir de este resultado fueron seleccionadas intencionalmente 22 empresas fabricantes de medicamentos, a las cuales se les aplicó el ICI, para validar su desempeño. Las empresas seleccionadas se localizaron en la ciudad de Bogotá y se trabajó con empresas que presentaron dife- rentes "capacidades de manufactura", tales como: Manufactura de productos antibióticos betalactámicos estériles; Manufactura de productos antibióticos betalactámicos no estériles; Manufactura de productos antibióticos no betalactámicos no estériles; Manufactura de productos hormonas no sexuales estériles / no estériles; Manufactura de productos hormonas sexuales no estériles; Manufactura de productos sin restricciones especiales en las áreas de manufactura; Manufactura de gases medicinales.

Una vez se realizaron las encuestas en cada una de las empresas, la información obtenida del ICI se organizó en bases de datos y se correlacionó con las capacidades de manufactura correspondientes. Las respuestas generadas sobre las preguntas de cada variable permitieron establecer un perfil de comportamiento, el cual fue confrontado con el referente, previamente definido como altamente competitivo; este resultado permitió establecer las capacidades tecnológicas para el conjunto de empresas estudiadas.

\section{RESULTADOS}

En la tabla 3 se presentan los resultados obtenidos en la aplicación del ICI en cada uno de los 22 laboratorios estudiados. 


\begin{tabular}{|c|c|c|c|c|c|c|c|c|c|c|}
\hline \multirow{22}{*}{ 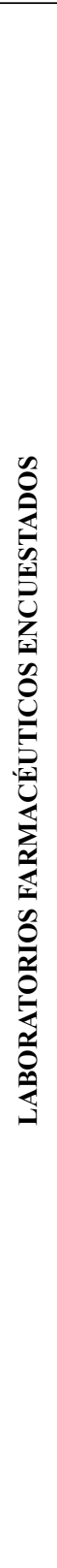 } & $\tilde{N}$ & 㫧 & $\stackrel{n}{\simeq}$ & $\hat{\theta}_{0}$ & $\begin{array}{l}+ \\
\text { in }\end{array}$ & $\begin{array}{l}0 \\
\ddot{q}\end{array}$ & $\begin{array}{l}0 \\
\sim\end{array}$ & $\begin{array}{l}\infty \\
\infty \\
\infty\end{array}$ & $\begin{array}{l}0 \\
\circ\end{array}$ & f \\
\hline & $\vec{\sim}$ & on & $\begin{array}{l}\text { m. } \\
\hat{n}^{n}\end{array}$ & $\begin{array}{l}\infty \\
8 \\
8\end{array}$ & $\begin{array}{l}\vec{j} \\
\text { in }\end{array}$ & $\begin{array}{l}0 \\
\ddot{q}\end{array}$ & $\begin{array}{l}0 \\
\approx\end{array}$ & $\begin{array}{l}\infty \\
\infty \\
\infty\end{array}$ & $\begin{array}{l}0 \\
9\end{array}$ & $\hat{d}$ \\
\hline & 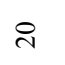 & $\begin{array}{l}\text { ra } \\
\text { of }\end{array}$ & $£$ & $\begin{array}{l}\vec{r} \\
\stackrel{2}{n}\end{array}$ & $\begin{array}{l}\vec{v} \\
\text { in }\end{array}$ & $\begin{array}{l}0 \\
\ddot{q}\end{array}$ & $\begin{array}{l}n \\
\tilde{a}\end{array}$ & $\vec{n}$ & $\begin{array}{l}0 \\
\stackrel{0}{=}\end{array}$ & $\begin{array}{l}\infty \\
\tilde{f}\end{array}$ \\
\hline & 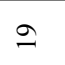 & on & $\begin{array}{l}n \\
\stackrel{n}{\simeq}\end{array}$ & $\hat{*}$ & $\begin{array}{l}\vec{j} \\
\text { in }\end{array}$ & $\begin{array}{l}0 \\
\dot{q}\end{array}$ & $\begin{array}{l}0 \\
\sim\end{array}$ & $\begin{array}{l}0 \\
\approx\end{array}$ & $\begin{array}{l}0 \\
9\end{array}$ & $\begin{array}{l}n \\
0 \\
0 \\
n\end{array}$ \\
\hline & $\stackrel{\infty}{-}$ & $\stackrel{m}{n}$ & $\begin{array}{l}0 \\
\stackrel{n}{n}\end{array}$ & $\vec{F}$ & 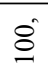 & $\begin{array}{l}0 \\
\stackrel{n}{n}\end{array}$ & $\stackrel{\infty}{\tilde{\lambda}}$ & 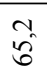 & $\stackrel{n}{=}$ & 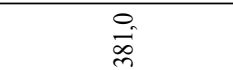 \\
\hline & $=$ & $\begin{array}{l}n \\
\text { ô }\end{array}$ & $\begin{array}{l}0 \\
i\end{array}$ & $\overrightarrow{6}$ & $\begin{array}{l}m \\
\stackrel{m}{ \pm}\end{array}$ & $\begin{array}{l}\infty \\
\infty \\
\infty\end{array}$ & 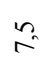 & $\begin{array}{l}0 \\
\text { of }\end{array}$ & $\stackrel{n}{=}$ & $\hat{n}$ \\
\hline & $\stackrel{0}{0}$ & $\stackrel{+}{\stackrel{N}{N}}$ & $\begin{array}{l}n \\
\cong\end{array}$ & $\begin{array}{l}n \\
\stackrel{n}{m}\end{array}$ & $\stackrel{\sigma_{0}}{\vec{N}}$ & $\begin{array}{l}\infty \\
\infty \\
\infty\end{array}$ & $\overbrace{0}^{n}$ & $\begin{array}{l}n \\
\tilde{n}\end{array}$ & $\stackrel{n}{=}$ & $\begin{array}{l}0 \\
0 \\
\infty\end{array}$ \\
\hline & 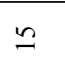 & ते & $\vec{f}$ & $\begin{array}{l}n \\
\dot{f}\end{array}$ & $\dot{8}$ & $\overrightarrow{\vec{\lambda}}$ & $\stackrel{n}{\simeq}$ & $\begin{array}{l}\hat{\sigma} \\
\vec{\gamma}\end{array}$ & $\hat{\curvearrowright}$ & त̂ \\
\hline & \pm & $\begin{array}{l}0 \\
\text { in }\end{array}$ & $\stackrel{0}{n}$ & 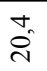 & 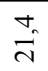 & $\ddot{0}$ & $\stackrel{0}{0}$ & $\frac{m}{n}$ & ते & $\begin{array}{l}0 \\
\text { हें }\end{array}$ \\
\hline & 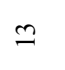 & $\frac{n}{m}$ & $\frac{n}{6}$ & $\hat{n}_{0}$ & $\begin{array}{l}\hat{\sigma} \\
\hat{~}\end{array}$ & $\overrightarrow{\vec{v}}$ & $\stackrel{n}{\simeq}$ & $\hat{\sigma}$ & $\hat{\text { oे }}$ & $\vec{F}$ \\
\hline & $\simeq$ & $\begin{array}{l}\infty \\
\stackrel{ \pm}{ \pm}\end{array}$ & $m_{\infty}^{m}$ & î & $\vec{\sigma}$ & $\begin{array}{l}0 \\
\stackrel{\sim}{\simeq}\end{array}$ & $\begin{array}{l}\infty \\
\infty \\
\infty\end{array}$ & $\overrightarrow{\tilde{n}}$ & $\begin{array}{l}0 \\
\stackrel{0}{2}\end{array}$ & $\hat{\tilde{\jmath}}$ \\
\hline & $=$ & $\stackrel{\sigma_{n}}{r}$ & ते & $\stackrel{m}{=}$ & बू. & $\begin{array}{l}0 \\
\stackrel{n}{n}\end{array}$ & $\stackrel{n}{\simeq}$ & $\hat{e_{0}}$ & $\hat{\vec{i}}$ & $\begin{array}{l}n \\
\stackrel{\sim}{\sim}\end{array}$ \\
\hline & $\circ$ & $\begin{array}{l}\stackrel{0}{\sim} \\
-1\end{array}$ & $\begin{array}{l}0 \\
i\end{array}$ & $\stackrel{m}{F}$ & $\because$ & $\ddot{O}_{0}$ & $\theta_{0}^{0}$ & $\hat{\text { ते }}$ & $\stackrel{n}{=}$ & $\stackrel{n}{\exists}$ \\
\hline & $a$ & $\overline{0}$ & $\stackrel{m}{m}$ & $\stackrel{\infty}{m}$ & $\hat{\sigma}$ & $\stackrel{0}{0}$ & ?ु? & $\begin{array}{l}0 \\
\hat{6}\end{array}$ & $\stackrel{n}{=}$ & ڤे \\
\hline & $\infty$ & $\stackrel{n}{m}$ & 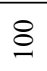 & $\vec{\nabla}$ & $\hat{\infty}_{\infty}^{m}$ & $\begin{array}{l}0 \\
\ddot{\sim}\end{array}$ & $\begin{array}{l}\infty \\
\infty \\
\infty\end{array}$ & $\begin{array}{l}0 \\
\infty \\
\infty\end{array}$ & $\hat{a}$ & 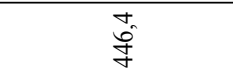 \\
\hline & $r$ & $\begin{array}{l}\stackrel{0}{f} \\
\dot{f}\end{array}$ & $\begin{array}{l}m \\
\infty \\
n\end{array}$ & $\begin{array}{l}\overrightarrow{+} \\
\dot{n}\end{array}$ & $\overrightarrow{m^{0}}$ & $\begin{array}{l}0 \\
n\end{array}$ & $\begin{array}{l}\text { mo } \\
\text { in }\end{array}$ & $\begin{array}{l}\infty \\
\infty \\
\infty\end{array}$ & $\stackrel{m}{=}$ & $\hat{a}$ \\
\hline & 0 & $\begin{array}{l}\dot{\forall} \\
\dot{m}\end{array}$ & $\begin{array}{l}0 \\
\stackrel{n}{v}\end{array}$ & $\begin{array}{l}\forall \\
\infty \\
-\end{array}$ & $\begin{array}{l}0 \\
\infty \\
\infty \\
\sim\end{array}$ & $\stackrel{0}{0}$ & $\begin{array}{l}0 \\
\text { in }\end{array}$ & $\stackrel{\infty}{\sim}$ & $\stackrel{0}{2}$ & $\begin{array}{l}\infty \\
\stackrel{ \pm}{ \pm}\end{array}$ \\
\hline & $n$ & $\begin{array}{l}n \\
\infty \\
0 \\
0\end{array}$ & $\stackrel{8}{ }$ & $\begin{array}{l}0 \\
\stackrel{+}{\pi}\end{array}$ & 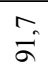 & $\begin{array}{l}0 \\
\text { in }\end{array}$ & $\begin{array}{l}\infty \\
\tilde{f}\end{array}$ & $\hat{\vec{q}}$ & $\stackrel{n}{=}$ & $\begin{array}{l}0 \\
\text { di } \\
\text { d }\end{array}$ \\
\hline & $\sigma$ & $\begin{array}{l}n \\
\infty \\
\infty\end{array}$ & $\begin{array}{l}0 \\
\text { in }\end{array}$ & $\begin{array}{l}\dot{r} \\
\stackrel{f}{f}\end{array}$ & $\stackrel{8}{\circ}$ & $\begin{array}{l}\infty \\
\infty \\
-1\end{array}$ & ?. & $\frac{\sim}{\infty}$ & $\begin{array}{l}0 \\
\stackrel{2}{2}\end{array}$ & $\begin{array}{l}\infty \\
\text { in }\end{array}$ \\
\hline & $n$ & $\begin{array}{l}n \\
\infty\end{array}$ & $\begin{array}{l}n \\
\text { ชิ }\end{array}$ & $\begin{array}{l}0 \\
8\end{array}$ & 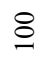 & $\begin{array}{l}\infty \\
\infty \\
\infty\end{array}$ & $\hat{n}_{0}$ & $\stackrel{+}{8}$ & $\begin{array}{l}\text { m. } \\
\text { }\end{array}$ & ָี \\
\hline & $N$ & $\hat{i}$ & กิ & $\underbrace{\infty}$ & : & $\begin{array}{l}\infty \\
\infty \\
\infty\end{array}$ & $n$ & 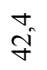 & ते & $\stackrel{n}{\stackrel{n}{\sim}}$ \\
\hline & - & $\hat{\sigma}_{0}$ & $\stackrel{m}{m}$ & $\stackrel{\circ}{i}$ & $\begin{array}{l}0 \\
\text { in }\end{array}$ & $\stackrel{0}{0}$ & $\overbrace{}^{2}$ & $\hat{F}$ & $\hat{\mathrm{i}}$ & $\hat{\hat{\Omega}}$ \\
\hline $\mathrm{S}$ & IdU & 离 & 诖 & $\stackrel{E}{F}$ & $\underbrace{\mathcal{O}}_{0}$ & $\frac{15}{2}$ & $\vec{\Delta}$ & $\underset{\simeq}{\mathbb{Z}}$ & 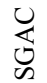 & $\begin{array}{l}\text { VDIOOTONOAL } \\
\text { VIDNGLAdWOJ }\end{array}$ \\
\hline
\end{tabular}


En el caso hipotético de una empresa que hubiese alcanzado los 100 puntos para cada una de las variables, el valor de la competencia tecnológica de la organización, asumiendo una contribución aditiva de las variables, correspondería a un total de 800 puntos.

Con la definición de este conjunto de variables para evaluar la competencia tecnológica se infiere un modelo descriptivo que puede facilitar la calificación del desempeño de una empresa del SFIP; este describe las organizaciones desde una perspectiva relacionada con sus procesos tecnológicos. Los datos de entrada se obtienen a través de la información obtenida de cada organización, la cual es codificada y valorada, para ser operada matemáticamente.

Se relacionaron las ocho variables definidas, de acuerdo con la ecuación 1:

\section{Ecuación 1.}

$$
\begin{aligned}
y= & \beta_{1} x_{1}+\beta_{2} x_{2}+\beta_{3} x_{3}+\beta_{4} x_{4}+\beta_{5} \\
& x_{5}+\beta_{6} x_{6}+\beta_{7} x_{7}+\beta_{8} x_{8}+\xi
\end{aligned}
$$

En donde:

$y$ : corresponde a competencia tecnológica; $x_{1}$ : integración con la cadena de abastecimiento; $x_{2}$ : escala de producción; $x_{3}$ : transferencia tecnológica; $x_{4}$ : perfil exportador; $x_{5}$ : innovación y desarrollo; $x_{6}$ : tecnologías de la información y la comunicación; $x_{7}$ : recursos humanos; $x_{8}$ : sistemas de aseguramiento de la calidad, sistemas de gestión ambiental, sistemas de seguridad industrial y de salud ocupacional.

$\xi=$ otras variables que pueden afectar la competencia tecnológica, no introducidos en el estudio y que pueden ser de carácter intrínseco o extrínseco de la organización.

$\beta=$ Define el efecto o peso que tiene cada variable sobre la competencia tecnológica.

Para la aplicación de esta metodología, se asume una ponderación igual para todas las variables sobre el resultado esperado en la variable respuesta, " $y$ ", lo que da lugar a la eliminación del factor $\beta$, siguiendo así el modelo, la forma de la ecuación 2. Esta consideración solamente es válida para el momento en el que se realiza el estudio, por tratarse de un estudio de tipo transversal descriptivo.

\section{Ecuación 2.}

$$
\begin{gathered}
y=x_{1}+x_{2}+x_{3}+x_{4}+x_{5}+x_{6}+x_{7}+ \\
x_{8}+\xi
\end{gathered}
$$


La aplicación de la ecuación 2 sobre los resultados obtenidos en el estudio, conllevan un estándar teórico de 800 puntos, definido como el comportamiento más competitivo en una organización. En la figura 2 se repre- senta el resultado de la aplicación de esta propuesta metodológica y la comparación de resultados entre las empresas estudiadas, en donde ninguna organización de la muestra superó un valor de 500 puntos.

Figura 2. Aplicación del modelo de competencia tecnológica para las organizaciones del SFIP de medicamentos ubicadas en Bogotá

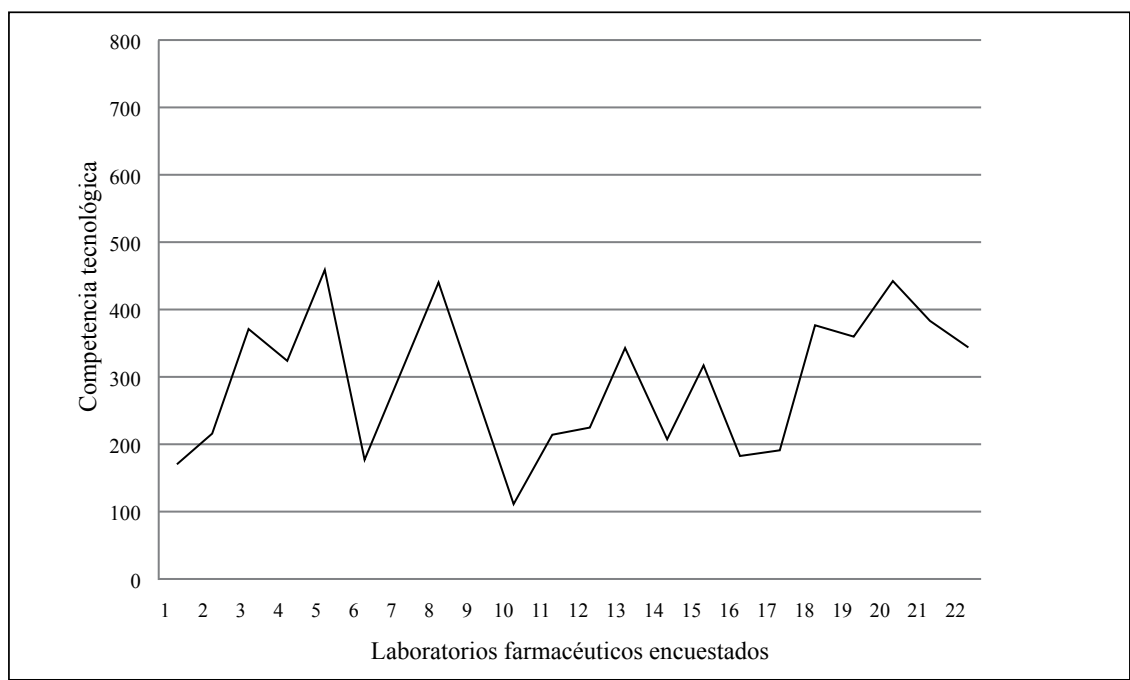

Fuente: elaboración propia.

\section{DISCUSIÓN}

Con la información generada mediante esta alternativa metodológica fue posible identificar para las organizaciones estudiadas, cuáles presentaron mayores valores de desempeño para cada una de las variables.

En el caso que se pretendan estudiar los comportamientos para cada una de las variables, independientes de la organización con un interés hacia una caracterización sectorial; con esta metodología es posible presentar los resultados más altos y más bajos obtenidos para cada variable, expresados porcentualmente en un gráfico radial, con el fin de comparar el comportamiento de las variables en el SFIP, ver figura 3. 
Figura 3. Comparación de resultados obtenidos entre las empresas con mayor y menor desempeño frente a las variables estudiadas

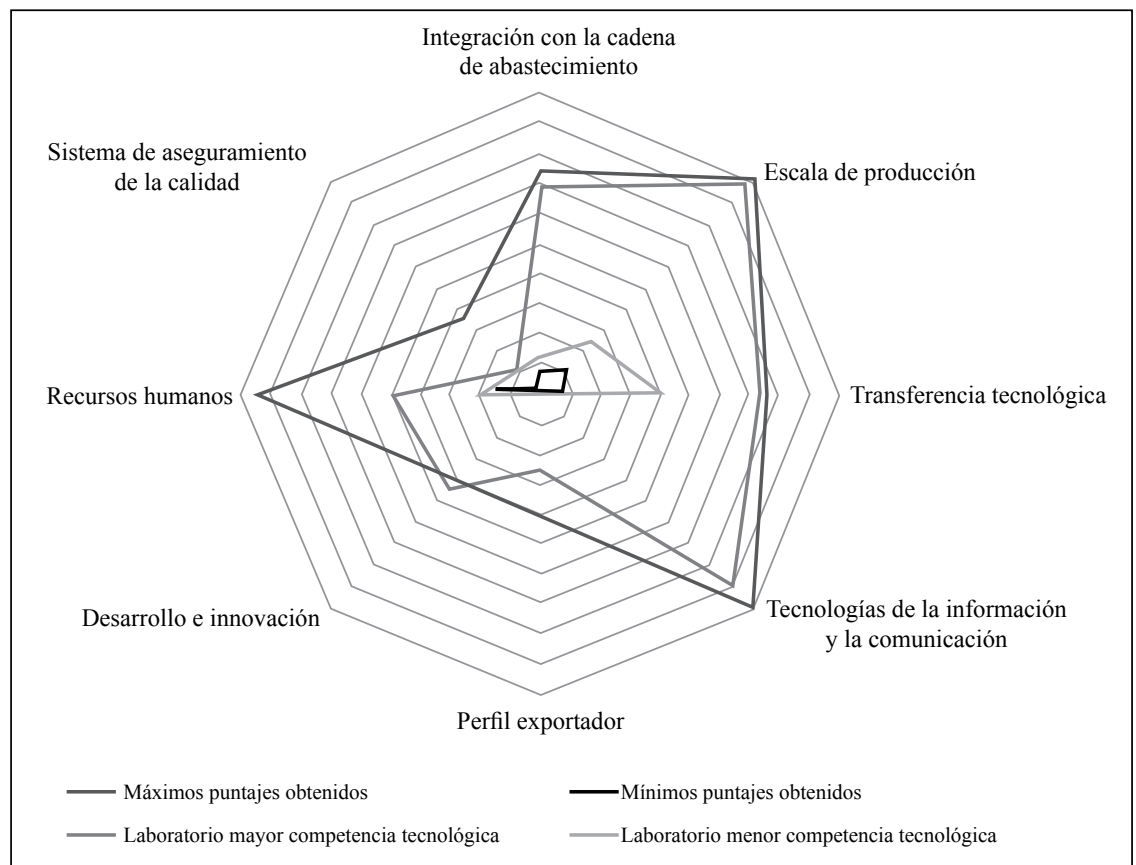

Fuente: elaboración propia.

Con la aplicación del ICI sobre las empresas estudiadas y habiendo definido que el $100 \%$, corresponde a un comportamiento teórico ideal o más competitivo, los resultados encontrados en las 22 empresas bajo estudio, presentaron un bajo desempeño de todas las organizaciones evaluadas frente a las variables relacionadas con Desarrollo e innovación, Sistemas de aseguramiento de la calidad y Perfil exportador. Este comportamiento puede ser determinante en un ámbito de globalización del sector y en la intención de mejorar el acceso de medicamentos de fabricación nacional a mercados externos.

Los hallazgos permitieron detectar diferencias entre el comportamiento teórico y el que desarrollan las organizaciones estudiadas. Los casos de cada organización dan lugar a descripciones más precisas. En el caso de la figura 3 , se observa que el laboratorio farmacéutico que obtuvo el mejor resultado al calcular la competencia tecnológica, muestra un bajo puntaje para las variables de 
perfil exportador, desarrollo e innovación, sistemas de calidad de producto, proceso y personal, y recursos humanos. Pero esta misma organización presenta un comportamiento de asociación con sus proveedores, una escala de producción competitiva, el desarrollado de varias actividades de transferencia tecnológica en los últimos cinco años, y ha integrado las tecnologías que permiten un manejo eficiente de la información al interior de la empresa.

Este resultado demuestra para este laboratorio farmacéutico, que ha desarrollado procesos de transferencia tecnológica para optimizar sus procesos y por ende sus productos. Sin embargo, la debilidad identificada en el comportamiento de esta organización del SFIP, es su poca capacidad para introducir productos en mercados internacionales, confirmado por el resultado en la evaluación de las variables diseño e innovación y sistemas de aseguramiento de la calidad. Esta descripción, asociada con el conocimiento de las capacidades de manufactura instaladas permitió realizar un análisis detallado de una organización del SFIP de medicamentos, desde una perspectiva tecnológica.

La metodología propuesta se presenta como una herramienta, con la cual no se contaba en el ámbito de la Industria Farmacéutica Colombiana, para evaluar el desempeño de las organizaciones del SFIP de medicamentos desde una perspectiva tecnológica; permite el análisis y descripción de desempeño y el reconocimiento de debilidades y oportunidades de mejoramiento, para una empresa.

Es posible a través de su aplicación representar la interacción entre variables, si bien el resultado obtenido no ofrece un curso de acción, si permite pronosticar el comportamiento dentro de la organización y eventuales medidas a seguir para alcanzar los desempeños más competitivos de estas variables. Esta herramienta es un comienzo para facilitar el análisis, el estudio y la descripción de las organizaciones de este sector, desde la perspectiva de los procesos de transformación de materiales. Algunos estudios (Tseng \& Pai, 2013) han concentrado su atención en la evaluación del conocimiento que posee la organización en el desarrollo de competencia tecnológica, pero su alcance es limitado, al no tener en cuenta otros elementos como los sugeridos en esta investigación.

La propuesta metodológica permite encontrar un esquema de evaluación de la competencia tecnológica de cada empresa productora de medicamentos, la cual sirve para 
aproximarse a un modelo de comportamiento del SFIP, en este caso para la ciudad de Bogotá.

Sin embargo, se evidenciaron algunas restricciones para la metodología que definen sus límites y alcances, además, la inclusión del factor " $\xi$ " permite considerar aspectos no relacionados con las ocho variables estudiadas, pero que pueden afectar la competencia tecnológica.

Estos aspectos que no fueron estudiados pueden ser de dos tipos:

- Los factores que afectan la competencia tecnológica, vinculados a aspectos financieros, políticos, administrativos y otro tipo de variables intrínsecas en el manejo de la organización.

- Los factores que afectan la competencia tecnológica, ligados a aspectos políticos, administrativos y tributarios, de carácter externo a la organización y dependientes de Políticas de Estado.

Los resultados obtenidos al aplicar esta propuesta a un laboratorio productor de medicamentos contribuyeron para la evaluación del estado de la organización, en relación con aspectos propios de las operaciones productivas. La identificación de diferencias entre comportamientos reconocidos como altamente competitivos y el desempeño de cada empresa, muestra una brecha asociada a los procesos de transformación de materiales que se dan en la organización, los cuales deben ser evaluados adicionalmente de manera particular, en el marco de las políticas empresariales, el estado financiero, la misión y la visión de esta.

\section{CONCLUSIONES}

Se construyó una propuesta metodológica original para evaluar la competencia tecnológica de las organizaciones del SFIP de medicamentos; se trata de una herramienta que facilita la descripción de las organizaciones, y permite realizar un estudio comparativo de las empresas en forma objetiva, establecer debilidades y fortalezas en relación con el entorno. Su aplicación y evaluación resultó ser de utilidad para el estudio del sector; los resultados obtenidos en el desafío con la muestra de estudio mostraron un bajo resultado general de las organizaciones frente a los comportamientos identificados como altamente competitivos.

El modelo definido como un comportamiento ideal u óptimo al ser utilizado como referente con los resultados obtenidos con los laboratorios farmacéuticos que participaron del estudio, ha permitido establecer fortalezas, debilidades y oportunidades 
de mejora de estas organizaciones $\mathrm{y}$ una visión del estado actual del sector a nivel distrital, que al concentrar la mayor población de productores de medicamentos del país, se constituye en una buena aproximación al ámbito nacional.

\section{AGRADECIMIENTOS}

Los autores agradecen a la Universidad Nacional de Colombia. Dirección de Investigación Sede Bogotá. Proyectos: "Evaluación del impacto de la logística de materiales del sector farmacéutico industrial productivo (SFIP), asociada al desarrollo tecnológico de sus actuales procesos de transformación" Fase I y Fase II.

\section{REFERENCIAS}

Achilladelis, B., \&Antonakis, N.(2001). The dynamics of technological innovation: the case of the pharmaceutical industry. Research Policy. April; 30(4), 535-588.

Ala, M. (2013). A firm-level analysis of the vulnerability of the Bangladeshi pharmaceutical industry to the TRIPS agreement: Implications for R\&D capability and technology transfer. Procedia Economics and Finances. June; (5) 30-39.

Albis, N. (2006). Redes de Innovación en la industria manufacturera 1989 - 1999. Tesis de Maestría en Ciencias Económi- cas. Bogotá, Colombia: Universidad Nacional de Colombia.

Attridge, J., \& Preker, A. (2005). Improving access to medicines in developing countries: Application of new institutional economics to theanalysis ofmanufacturingand distribution issues. Washington DC, USA: Health Nutrition and Population (HNP). The World Bank; March p. 9.

Bessen, J. (2009). Estimates of patent rents from firm market value. Research Policy. Dec; 38 (10), 1604-1616.

Cohan, P. (1999). Los Lideres en tecnología. Innovación: el camino que llevo al éxito a las empresas tecnológicas más importantes de Estados Unidos. México: Prentice Hall; p. 10.

De Carolis, D.M. (2003). Competencies and imitability in the pharmaceutical industry: an analysis of their relationship with firm performance. Journal of Management. January; 29 (1) 27-50.

Departamento Nacional de Planeación. (2008). Documento Conpes 3527. Política Nacional de Competitividad y Productividad. [Internet]. Bogotá, Colombia: Consejo Nacional de Política Económica y Social. [Actualizado Junio 2008; citado Diciembre 2008]. Recuperado de https:// www.dnp.gov.co/Portals/0/archivos/documentos/Subdireccion/Conpes/3527.pdf 
Gallo, J. Estudio de la relación proveedor-productor en la gestión de Materiales del sector farmacéutico industrial productivo (SFIP) de la ciudad de Bogotá. Tesis de Maestría en Ciencias Farmacéuticas. Bogotá, Colombia. Universidad Nacional de Colombia.

Gallo,J., Vallejo, B.,\&Plazas, C.(2010). La Capacidad de Manufactura como un Indicador de Desarrollo Tecnológico en el Sector Farmacéutico Industrial. Ingeniería e Investigación. 30(1). 112-117.

Gnyawali, D., \& Park, B. J. (2009). Co-opetition and technological innovation in small and medium- size enterprises: a multilevel conceptual model. Journal of small business management. July; 47 (3) 308 - 330

González, M. (2004). Determining the importance of the supplier selection process in manufacturing: a case study. International Journal of Physical Distribution \& Logistics Management. June; 34 (6): 492-504.

Kaplan, W., \& Laing, R. (2005). Local production of pharmaceuticals: industrial policy and access to medicines: An overview of key concepts, issues and opportunities for future research. Washington DC, USA: Health Nutrition and Population (HNP). The World Bank. January. p. 2-7.

Keld, L. (1996). Horizontal diversification in the Danish national system of innovation: the case of pharmaceuticals. Research Policy 25(7) 1121-1137.

Meijboom, B., \& Obel, B. (2007). Tactical coordination in a multilocation and multistage operations structure: a model and a pharmaceutical company case. Omega. Jun; 35(3) 258-273.

Ministerio de la Protección Social. (2008). Resolución 3028 de 2008 por la cual se definen las áreas técnicas de producción de los establecimientos farmacéuticos y se establecen otras disposiciones. [Internet]. Bogotá, Colombia: Ministerio de la Protección Social. Disponible https://www. minsalud.gov.co/Normatividad Nuevo/RESOLUCIÓN 3028 DE 2008.pdf

Mora, C. (2003). Interrelaciones entre las empresas multinacionales $y$ las firmas locales farmacéuticas. Impacto en el desarrollo industrial de este sector en Colombia. Tesis de Maestría en Administración. Bogotá, Colombia: Universidad Nacional de Colombia.

Organización Mundial de la Salud. (1999). Comité de expertos de la OMS en especificaciones para las preparaciones farmacéuticas Informe 35.Ginebra. OMS.

Rodríguez, D., \& Julio, M. (2006). La dinámica compleja de la innovación tecnológica. Visión Hiper 666. Sumergiéndose en las interrelaciones, la incertidumbre, el 
caos y las emergencias. Edición Facultad de Ingeniería. Universidad Nacional de Colombia;

Ryan, M., \& Shanebrook, J. (2009).

Establishing globally-competitive pharmaceutical and bio-medical technology industries in Jordan - assessment of business strategies and the enabling environment. [Internet]. Washington, D.C, USA: International Intellectual Property Institute. 2004. Disponible en $\mathrm{http}: / / \mathrm{www}$. iipi. or/reports/Jordan_Report.pdf

Simango,C.(2000). Corporatestrategy R\&D and technology transfer in the European pharmaceutical industry: research findings European Pharmaceutical industry. European Business Review 12(1) 28-33.

Tseng, C., \& Pai, D. (2013). Knowledge search, spillover and creation capability in India's pharmaceutical industry. Technology Analysis and Strategic Management. October; 6 (2): 207-222

Vallejo, B., \& Torres, N. (2007). Desarrollo Tecnológico del Sector Farmacéutico Industrial Asociado a Procesos de Transformación de Materiales. Vitae. Jul-Dec; 14(2), 59-70.

World Health Organization. (2007). Quality assurance of pharmaceuticals: A compendium of guidelines and related materials. Volume 2. 2nd Updated edition. Geneva, Switzerland: WHO.

Zucker, L.G., \& Darby, M. R. (1997). Present at the biotechnological revolution: transformation of technological identity for a large incumbent pharmaceutical firm. Research Policy. Dec; 26 (4): 429-446. 